\title{
Super-Resolution Electron Microscopy using Multi-Resolution Data Fusion
}

\author{
Charles A. Bouman
}

Showalter Professor, Electrical and Computer Engineering, Purdue University, West Lafayette, IN.

Scanning transmission electron microscopes are widely used for characterization of samples at the nanometer scale. However, raster scanning an electron beam across a large field of view is time consuming and can often damage the sample. In fact, the sum total of all electron microscopy (EM) data collected to date, represents less than a cubic millimeter of material [1]. Consequently, there is an enormous demand in the materials and biological sciences to image at greater speed and lower dosage, while maintaining resolution. Traditional EM imaging based on homogeneous raster-order scanning severely limits the volume of high-resolution data that can be collected, and presents a fundamental limitation to understanding physical processes such as material deformation, crack propagation, and pyrolysis. For these reasons, there is growing interest in reconstructing full-resolution images from sparsely-sampled $[2,4]$ and low-resolution images [3].

In this talk, we introduce a novel multi-resolution data fusion (MDF) method [5] for super-resolution computational EM. Our method combines innovative data acquisition with novel algorithmic techniques to dramatically improve the resolution/volume/speed trade-off. Our method depends on a Bayesian framework for the fusion of low- and high-resolution data without the need for training. The key to our approach is to collect the entire sample at low resolution, while simultaneously collecting a small fraction of data at high resolution. The high-resolution measurements are then used to create a materialspecific patch-library that is used within the "plug-and-play" (P\&P) framework [4] to dramatically improve super-resolution of the low-resolution data.

The P\&P framework is based on the alternating direction method of multipliers (ADMM) and decouples the forward model and the prior terms in the maximum a posteriori cost function. This results in an algorithm that involves repeated application of two steps: an inversion step only dependent on the forward model, and a denoising step only dependent on the image prior model. The P\&P takes ADMM one step further by replacing the prior model optimization by a denoising operator of choice.

We present results using FEI electron microscope data that demonstrate super-resolution factors of $4 x$, $8 x$, and 16x, while substantially maintaining high image quality and reducing dosage [6]. 


\section{References:}

[1] D.B.Williams and C.B. Carter. Transmission electron microscopy, Springer (1996) pp. 3.

[2] C. Guillemot and O. Le Meur. Signal Processing Magazine, IEEE, 31(1) (2014), pp. 127.

[3] S.C. Park, M.K. Park, and M.G. Kang. Signal Processing Magazine, IEEE, 20(3) (2003), pp. 21.

[4] S. Sreehari, S.V. Venkatakrishnan, B. Wohlberg, G.T. Buzzard, L.F. Drummy, J.P. Simmons, and C.A. Bouman. Transactions on Computational Imaging, IEEE, 2(4) (2016), pp. 408.

[5] S. Sreehari, S.V. Venkatakrishnan, K.L. Bouman, J.P. Simmons, L.F. Drummy, and C.A. Bouman. arXiv:1612.00874 (2016).

[6] This work was supported by AFOSR/MURI grant \#FA9550-12-1-0458, by UES Inc.

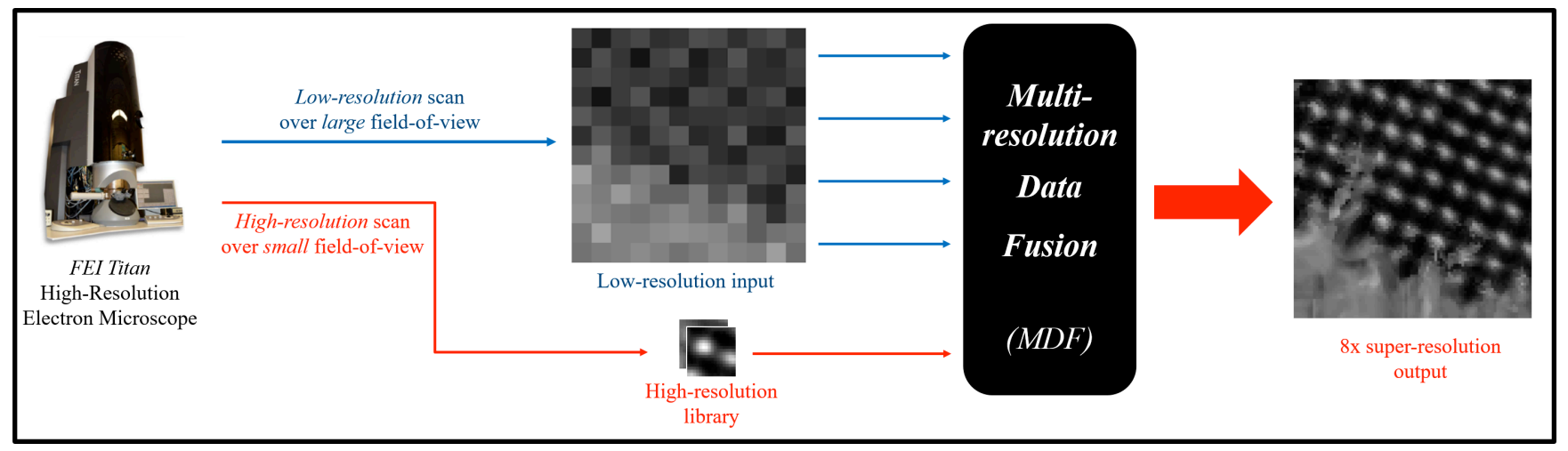

Figure 1. Illustration of multi-resolution data fusion (MDF) for 8x super-resolution of a TEM image of gold atoms.

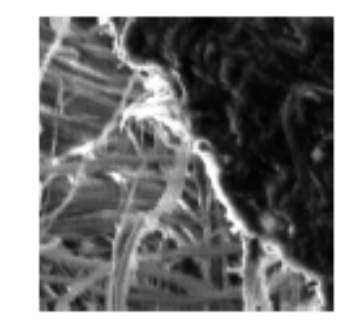

(a) A portion of the library image

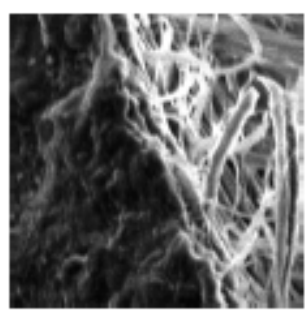

(b) Ground truth

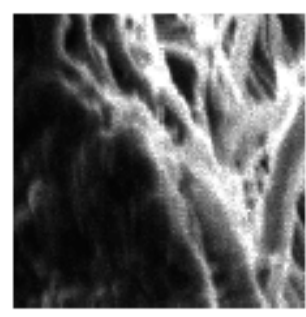

(c) Ground truth (zoomed)

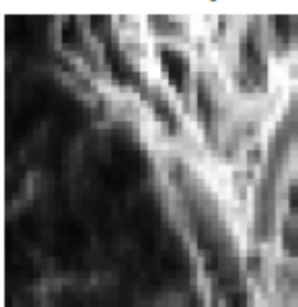

(d) Low-resolution input

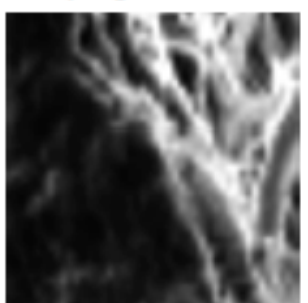

(e) $4 \mathrm{x}$ cubic inkerpolation

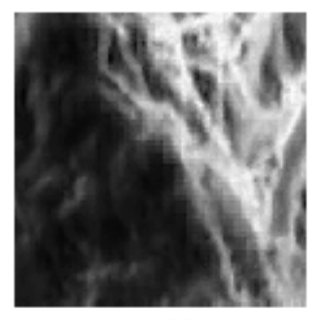

(f) $4 \pi$ SISR

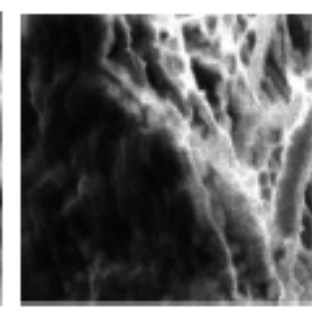

(g) 4 M MDF (proposed algorithm)

Figure 2. 4x super-resolution of a $100 \times 100$ SEM image of surface crack in the shell of the marine mollusk Hinea brasiliana. We compare our result against Technion's single image super-resolution (SISR) algorithm, and observe that our MDF algorithm lacks block artifacts and jaggies. 